\title{
Graph Rewriting for Enhanced Universal Dependencies
}

\author{
Bruno Guillaume \\ Université de Lorraine, CNRS, \\ Inria, LORIA, Nancy, France \\ bruno.guillaumeeinria.fr
}

\author{
Guy Perrier \\ Université de Lorraine, CNRS, \\ Inria, LORIA, Nancy, France \\ guy perrieraloria.fr
}

\begin{abstract}
This paper describes a system proposed for the IWPT 2021 Shared Task on Parsing into Enhanced Universal Dependencies (EUD). We propose a Graph Rewriting based system for computing Enhanced Universal Dependencies, given the Basic Universal Dependencies (UD).
\end{abstract}

\section{Introduction}

The IWPT 2021 Shared Task on Parsing into Enhanced Universal Dependencies (Bouma et al., 2021 ) is a second edition of an equivalent shared task in 2020 (Bouma et al., 2020). The goal of the shared task is to produce EUD (Schuster and Manning, 2016), with several new annotation layers expressed on top of UD annotations (Nivre et al., 2020).

In the previous shared task, there were two kinds of approaches: producing EUD annotation from raw text with machine learning methods or producing EUD from UD with a rule-based approach (with or without some learning to optimize rule usage). Like (Heinecke, 2020) or (Dehouck et al., 2020), our proposal corresponds to the second approach: we used an existing tool for producing UD annotations and work only on the conversion from UD to EUD. Unlike other rule-based approaches, we used GREW, a generic Graph Rewriting tool (Bonfante et al., 2018), in order to describe the rules for enhancement.

Another specificity of our work is that we primarily design our rules by following the guidelines. Even if, in a secondary step and in the context of the shared task, we adapt the system to the corpora which diverge from the guidelines (section 2.6), we can easily provide a system closer to the guidelines, adpatable to languages specificities.

Our system achieved 81.58 ELAS score on the task, starting from UDPIPE annotation with an
LAS of 85.77. In the paper, we present the system, analyse the results and make some in-depth analysis on French and English of discrepancies between GOLD $\operatorname{LU}_{E U D}$ data and the output of our system starting $\mathrm{GOLD}_{U D}$ annotations.

\section{Description of the system}

\subsection{Parsing to UD}

For this shared-task, we used the UDPIPE2 (Straka et al., 2016) through the online service ${ }^{1}$ to produce the UD annotation of the data. The models used for each language are trained in UD version 2.6 on the following corpora: Arabic-PADT, Bulgarian-BTB, Czech-PDT, English-EWT, Estonian-EDT, FinnishTDT, French-GSD, Italian-ISDT, LithuanianALKSNIS, Dutch-Alpino, Polish-PDB, SlovakSNK, Swedish-Talbanken, Tamil-TTB, Ukrainian$\mathrm{IU}^{2}$.

\subsection{GREW}

The transformation UD to EUD is described with the graph rewriting tool $\mathrm{GREW}^{3}$. Each rule is defined by a pattern and a set of commands describing how to modify the graph. A dedicated strategy mechanism allows for controlling rules applications (in which order subsets of rules must be applied and how they must be iterated). A global transformation system (rules and strategies) is called a Graph Rewriting System (GRS).

\subsection{Representation of EUD annotations}

We use here the convention already adopted in the Deep-Sequoia project (Candito et al., 2014), in which dependencies are drawn in black when the

\footnotetext{
${ }^{1}$ https://lindat.mff.cuni.cz/services/ udpipe/

${ }^{2}$ The only modification done was on the Czech output where 7 obvious errors on the lemmatisation makes the conversion producing non valid data.

${ }^{3}$ https://grew.fr/
} 
relation exists both in UD layer and EUD layer, in red when they are present in UD layer only and in blue (and below) when they are present in EUD layer only (see Figure 1). This prevents from having two relations when both layers are identical and make figures easier to read.

\subsection{From UD to EUD}

Our goal was to design a GRS, following the EUD guidelines, to convert the UD annotations into EUD annotations, but we observed that the conversion system has to be adapted to each language and to some particular annotation choices.

As the rules of a GRS are organized in strategies, this adaptation is done by activating or not the applications of subsets of rules in the definition of the main strategy of a generic GRS. The rules are freely available . $^{4}$

\subsubsection{The six types of enhancement}

The EUD guidelines identify 6 types of enhancements, and for each type, we have designed a subset of rules to achieve it. We briefly describe the main features of each subset.

Empty nodes for elided predicates Predicate elision is detected thanks to the presence of orphan dependencies. They are mainly found in coordinations and sometimes with parataxis relations. Figure 1 shows the UD annotation of a sentence with ellipsis 5 .

First, a null node $\mathrm{N}$ is created and a dependency $\mathrm{N}-\mathrm{obj} \rightarrow$ trees is introduced from $\mathrm{N}$ to the head of the second conjunct trees. The label $\circ \mathrm{b} j$ is determined by a parallelism with the dependency sold -obj $\rightarrow$ strawberries ${ }^{6}$. The dependency trees $-\mathrm{CC} \rightarrow$ and is also raised to N.

In a second stage, all orphan dependencies from the head of the second conjunct are transformed into dependencies from the null node. The labels of the new dependencies are determined from the context. In our example, the dependency trees -orphan $\rightarrow$ winter is transformed into the dependency $\mathrm{N}-\mathrm{Obl} \rightarrow$ winter, because $\mathrm{N}$ is a verb, winter a noun with a case dependent.

\footnotetext{
${ }^{4}$ https://gitlab.inria.fr/grew/udtoeud

${ }^{5}$ All examples are extracted form the corpora used in the task. Due to lack of space, expressions in square brackets, which are not essential to our purpose, are skipped in the annotation.

${ }^{6}$ Even if the subject is also a candidate, we always favour the object in this case.
}

Propagation of incoming dependencies to conjuncts The propagation of incoming dependencies to the conjuncts of a coordination is systematical. The only difficulty concerns modifiers: if a word $\mathrm{H}$ is modified by a coordination, the label of the dependency from $\mathrm{H}$ to the head of the first conjunct may need to be changed depending of the POS of the second conjunct. In Figure 2, the dependency come -obl $\rightarrow$ parents has to be propagated to the head of the other conjunct, the adverb separately. Because of this POS, the label has to be changed and the dependency becomes come -advmod $\rightarrow$ separately.

Many gold corpora of the task do not take into account differences of POS between the conjunct heads and propagate the incoming dependencies without changing their labels.

\section{Propagation of outgoing dependencies from con-} juncts The main problem for this enhancement comes from the ambiguity of the UD annotation schema. It is not possible to distinguish a left dependency on a coordination from a left dependency on the first conjunct of the coordination because both are attached to the head of the first conjunct.

But, it is necessary to remove the ambiguity in order to know if one should propagate a left dependency. This is more or less easy depending on the type of the dependency. In Figure 3, the nsubj and cop dependencies on the noun acteur must be propagated on the head of the second conjunct protagoniste. It is easy to design a specific rule for each type of dependency to perform the propagation, what we have done. But, if the dependency is an advmod dependency, there is no general criterion for removing the ambiguity. The dependency on souvent should be propagated, but not the dependencies on $\mathrm{n}^{\prime}$ and pas. This depends on the modifier adverb but also on the context. This is a point where our rule-based approach marks its limits compared to learning approaches. Of course, the answer depends strongly on the language and we will see how to take into account the specificity of each language in subsection 2.5.

For right dependencies, there is no ambiguity, because a right dependent on the first conjunct that follow all conjuncts is necessarily a dependent on the coordination.

Additional subject relations for control and raising constructions Raising and control verbs take an infinitive as a xcomp dependent and the en- 


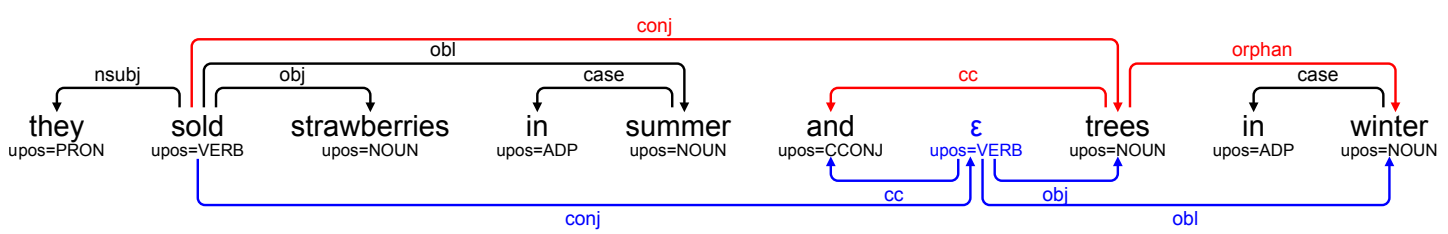

Figure 1: They sold strawberries in summer [,] and [Christmas] trees in [the] winter

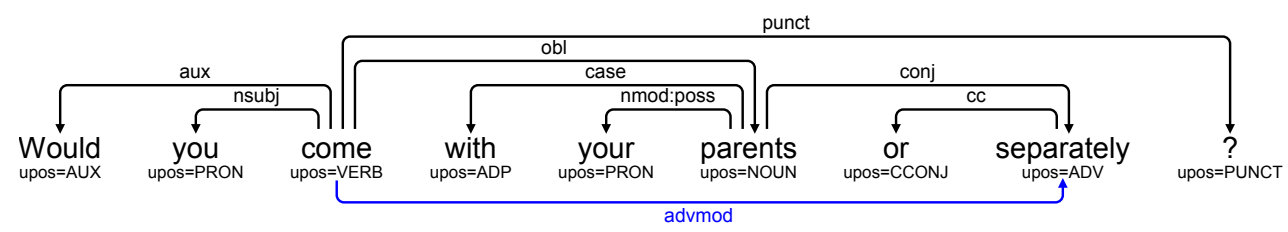

Figure 2: Would you come with your parents or separately?

hanced subject of the infinitive is either the subject, the direct object or the indirect object of the main verb, if that argument exists. The choice between the three possibilities depends on the lexical information about the raising or control verbs. In our approach, this means that we need lexicons per language. Most often, we do not have such lexicons and choose the subject of the main verb as the subject of the infinitive, as this is the most likely.

\section{Coreference in relative clause constructions}

The relative clause enhancement adds ref dependencies from the antecedent to the relative pronoun and all dependencies targeting the relative pronoun are moved to the antecedent.

In Figure 4, the sentence contains two relative pronouns, dont and qui. Let us focus on qui. First, rules make an upward path from the relative pronoun by following the dependencies until finding an acl: relcl dependency. In the example, this requires crossing a dependency nsubj and then conj. As soon as the dependency acl $: r e l c l$ is reached, it is possible to add the ref dependency, because its source is known, it is the source of the dependency acl:relcl and the antecedent of the relative pronoun, and the target of the dependency as well, the relative pronoun, which is kept in memory. In a second stage, all dependencies targeting the relative pronoun are moved to the antecedent. In the example, the dependency nécessite - nsubj $\rightarrow$ qui is transformed into the dependency nécessite -nsubj $\rightarrow$ entreprise.
Modifier labels that contain the preposition or other case-marking information With regard to this enhancement, we strictly follow the guidelines: if a case or mark dependent on a modifier is a multiword expression, we add the form of the expression to the dependency representing the modification; if it is a single word, we add the lemma of the word.

The guidelines don't cover the case of several case or mark dependencies having the same source. In Figure 5, the conjunction because and the preposition in both depend on the same governor zone (dependencies in orange). We decide to add the outermost dependent lemma to the modifier dependency feel $-\operatorname{advcl} \rightarrow$ zone because it is related to this dependency, whereas in has no relationship to it.

If the two candidate dependents are consecutive, we consider them as a single multiword and add the concatenation of their lemmas to the modifier dependency.

\subsubsection{Rule ordering}

The six types of enhancements are not totally independent, some of them interact, so the order of application between the six corresponding subsets of rules is not neutral. Figure 1 shows an interaction between the subset implementing ellipsis processing and the subset implementing the propagation of outgoing dependencies of a coordination. If we apply the latter first, it is not possible to propagate the dependency sold - nsub $j \rightarrow$ they to the second conjunct of the coordination because its verb is elided. We must apply first the subset creating a null node representing this verb. 


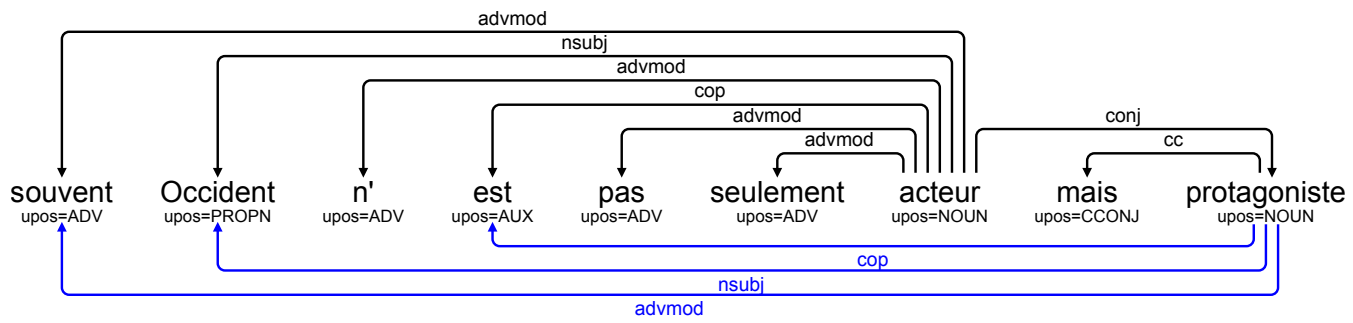

Figure 3: souvent [, l'] Occident n' est pas seulement [1'] acteur [,] mais [aussi le] protagoniste [des violations des droits de l' homme] (often[, the] West is not only [the] actor[,] but [also the] protagonist [of human rights violations])

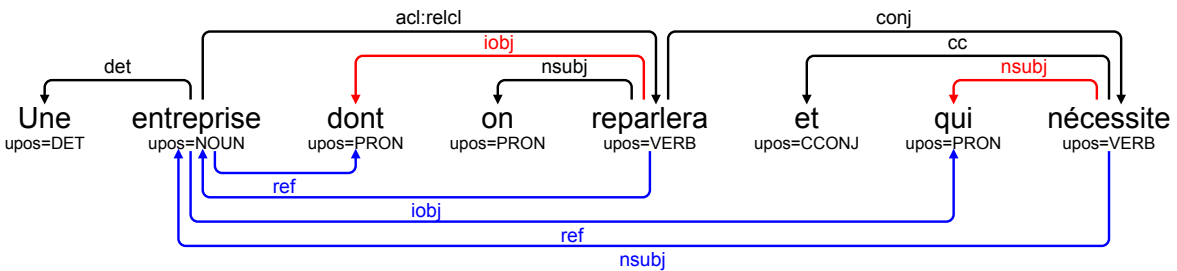

Figure 4: Une entreprise dont on reparlera et qui nécessite [un budget important] (A business that will be talked about again and that requires [a large budget])

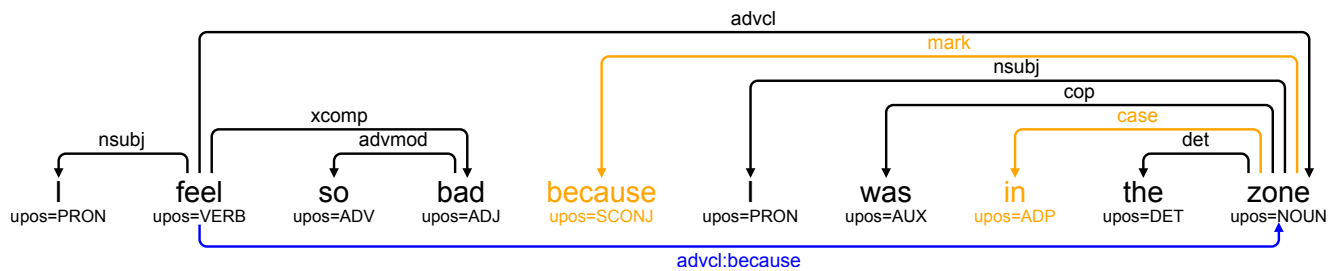

Figure 5: I feel so bad because I was [so] in the zone [that I didn't even get her name] 
The subset of rules adding case or mark information to modifier dependencies must be applied at the end because it does not create any possibility to apply another subset later.

The order between the application of the five other subsets is relatively free. Figure 4 shows an example of interaction between the subset relating to relative clauses and the subset implementing the propagation of incoming dependencies of a coordination. We applied the former subset first and to apply the second subset, we only need to propagate the dependency entreprise -acl: $\mathrm{relcl} \rightarrow$ reparlera to the second conjunct of the coordination. But there is no problem to reverse the order between the two subsets.

Even if the order chosen between the 6 subsets aims at minimizing the number of rule applications, we cannot avoid applying some subsets a second time. Figure 6 shows an example of repetition in the subset application. To add the dependency build - nsubj $\rightarrow I$, we need first to apply the subset related to control verbs. We obtain the dependency sell - nsubj $\rightarrow I$. Then we apply the subset related to the propagation of outgoing dependencies of a coordination. We obtain the dependency use - nsub $j \rightarrow I$. Finally, we apply the subset relative to control verbs a second time and we obtain the last dependency build - nsubj $\rightarrow$ I.

All rules presented in this subsection constitute the generic GRS used to convert the UD annotation into the EUD for the 17 languages.

\subsection{Adaptation to the specificities of languages}

Rule packages are added to the generic GRS to express specificities of language groups. In order to be applied, they are inserted in the strategy at carefully chosen positions in the generic strategy.

In this way, strategies can be designed adapted to particular languages, by activating or not these new packages.

Now, let us examine which types of rules can be added to express specificities of certain languages.

Null Subject Languages Arabic, Bulgarian, Czech, Estonian, Finnish, Italian, Polish, Russian, Slovak, Tamil and Ukrainian are null subject languages. Their grammar permits verbs to lack an explicit subject. This can be a problem for the propagation of subjects of coordinated verbs.

Consider the Polish sentence "Moje gospodarstwo daje mi zabezpieczenie, mam gdzie wrócić
(My farm gives me security, I have a place to come back to)". The general rules of subject propagation will propagate the subject gospodarstwo from the verb daje to the coordinated verb mam, which is incorrect because mam is at first person and does not require any explicit subject. In order to avoid the propagation, a specific rule marks all first and second person finite verbs, so that they cannot receive a subject dependency. For the third person, there is an ambiguity. In the Polish sentence "Chtopiec wstaje, otwiera drzwi", there are two correct translations in English: "The boy gets up, opens the door" and "The boy gets up, he opens the door", because one can propagate the subject boy or not. We chose to propagate the subject, which means that in this case, there is no difference between null subject languages and others.

Case addition to the dependency labels for modifiers For case-based languages, the labels of the dependencies targeting modifiers are augmented with their cases. The rule package implementing this enhancement is trivial.

Left dependents of a coordination We designed rules to propagate the left dependents of a coordination by dependency types. As we said before, a left dependent on the first conjunct of a coordination is ambiguous: it can depend on the whole coordination or only on the first conjunct. In order to determine, for a given language if a given type of dependency must be propagated or not, we tested the two alternatives on the dev corpus of the shared task and keep the alternative yielding the highest score.

This method has important limits because it depends on the annotation of the gold corpus. Moreover it is very coarse; for a given dependency type, not all dependencies have the same behaviour: some must be propagated, others not. It would be necessary to refine the conversion rules but for that, we need linguistic knowledge about the concerned language.

Raising and control verbs The default rule we use is to consider that the subject of the raised or controlled verb is the subject of the main verb but this is not always true. A language-specific lexicon should indicate for each of these verbs which argument of the main verb is the subject of the raised or controlled verb. From the training and development corpora available for the task, we have created lexicons for a five languages: Dutch, English, French, 


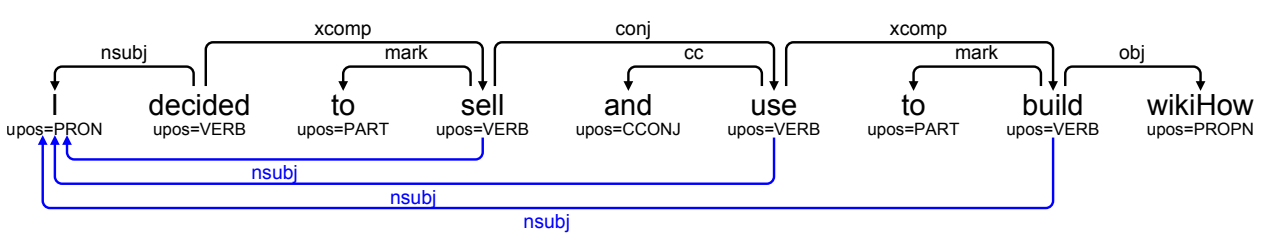

Figure 6: [So] I decided to sell [eHow] and use [the proceeds] to build wikiHow.

Italian and Polish.

\subsection{Adaptation to annotation choices}

Some annotators of the gold corpora do not strictly follow the guidelines. We have adapted our GRS to their choices on some very specific points.

Dependency label extension specific to one language In Dutch, enhancements for relative clauses distinguish antecedents of relative pronouns that play the deep role of subject and direct object in the relative clause with the extensions relsubj and relobj. We have designed specific rules to add this extension.

Enhancements partially taken into account We have taken into account the fact that enhancements are only partially achieved for Arabic, Bulgarian, Estonian, French, Russian and Tamil.

Coordinating conjunction raising For the Arabic, Dutch, English, Italian and Swedish treebanks, the names of coordinating conjunctions are added to the corresponding con $j$ dependencies, in the same way as for prepositions and subordinating conjunction. We have taken this into account even though it is not indicated in the guidelines.

Propagation of root dependencies According to the guidelines, root incoming dependencies of a coordination should be propagated like all incoming dependencies, but some treebanks do not and we take this into account.

The French and the Polish treebanks, the latter partially, not only add subjects for raising and control verbs, as mentioned in the guidelines, but add deep subjects for modifier infinitive and participial clauses. Since this goes beyond the guidelines, we do not consider these enhancements.

Table 1 summarizes the three kinds of adaptation to the different languages.

\section{Results}

Data we submit to the task, called GREW(UDPIPE), is the output of the application of a language spe-

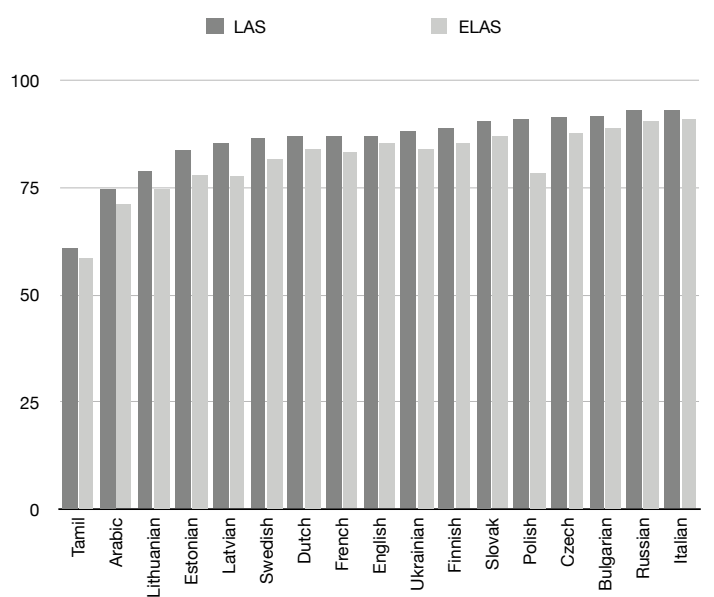

Figure 7: LAS of UDPIPE and ELAS of GREW(UDPIPE)

cific GRS to the output of UDPIPE. Figure 7 shows for each language, the LAS of UDPIPE and the ELAS of GREW(UDPIPE). We can observe that the final result highly depends on the UD annotation quality produced by UDPIPE.

In addition to the results provided by the orgnanisers, we make complementary analysis, focusing on the UD to EUD transformation. In Table 2, we report the ELAS score obtained by our system applied on the gold UD annotation as input. We also recall the same measure reported last year by two others systems (Dehouck et al., 2020; Heinecke, 2020). The score of our system is in most of the case between the two other proposal, closed to Dehouck's system except for Estonian and Swedish.

\section{Analysis of discrepancies with the gold annotation In French and English}

In order to better understand the behaviour of our GRS, we made some manual inspection of the difference between the annotation $\operatorname{GREW}\left(\mathrm{GOLD}_{U D}\right)$ and GOLD $\mathrm{EUD}_{D}$. We focused on the two languages for which we had the grammatical skills to analyze the discrepancies: French and English. 


\begin{tabular}{|l|c|c|c|c|c|c|c|c|c|c|c|c|c|c|c|c|c|}
\hline & ar & bg & cs & nl & en & et & fi & fr & it & lv & lt & pl & ru & sk & sv & ta & uk \\
\hline \hline null subject & $\mathrm{X}$ & $\mathrm{X}$ & $\mathrm{X}$ & & & $\mathrm{X}$ & $\mathrm{X}$ & & $\mathrm{X}$ & & & $\mathrm{X}$ & $\mathrm{X}$ & $\mathrm{X}$ & & $\mathrm{X}$ & $\mathrm{X}$ \\
\hline left dep propag & & & & & & & $\mathrm{X}$ & & & $\mathrm{X}$ & & & & & & & \\
\hline left aux dep propag & & & & & & & & & & & & $\mathrm{X}$ & & & & & \\
\hline left case dep propag & & & & & & & & & & $\mathrm{X}$ & & $\mathrm{X}$ & & & & & \\
\hline left cop dep propag & & & & & & & & & & $\mathrm{X}$ & & $\mathrm{X}$ & & & & & \\
\hline left mark dep propag & & & & & & & & & & $\mathrm{X}$ & & $\mathrm{X}$ & & & & & \\
\hline case raising & & & $\mathrm{X}$ & & & $\mathrm{X}$ & $\mathrm{X}$ & & & $\mathrm{X}$ & $\mathrm{X}$ & $\mathrm{X}$ & $\mathrm{X}$ & $\mathrm{X}$ & & $\mathrm{X}$ & $\mathrm{X}$ \\
\hline \hline subj control raising & & & & $\mathrm{X}$ & $\mathrm{X}$ & & & $\mathrm{X}$ & $\mathrm{X}$ & & & $\mathrm{X}$ & & & & & \\
\hline \hline specific extens & & & & $\mathrm{X}$ & & & & & & & & & & & & & \\
\hline partial enhancement & $\mathrm{X}$ & $\mathrm{X}$ & & & & $\mathrm{X}$ & & $\mathrm{X}$ & & & & & $\mathrm{X}$ & & & $\mathrm{X}$ & \\
\hline coord conj raising & & & & $\mathrm{X}$ & $\mathrm{X}$ & & & & $\mathrm{X}$ & & & & & & $\mathrm{X}$ & & \\
\hline root propagation & & & & & & & & & & & $\mathrm{X}$ & $\mathrm{X}$ & $\mathrm{X}$ & $\mathrm{X}$ & & $\mathrm{X}$ & $\mathrm{X}$ \\
\hline
\end{tabular}

Table 1: The three kinds of adaptation of the system. Top: adaptation to languages; middle: adding lexical information; bottom: adaptation to specific annotations observed in dev data.

\begin{tabular}{|c|c|c|c|}
\hline Language & Dehouck & Heinecke & Our \\
Shared task & 2020 & 2020 & 2021 \\
\hline Arabic & 98.8 & 95.2 & 98.5 \\
Bulgarian & 98.6 & 97.8 & 97.6 \\
Czech & 97.9 & 94.7 & 97.6 \\
Dutch & 98.9 & 94.4 & 97.6 \\
English & 99.5 & 98.0 & 99.0 \\
Estonian & 99.2 & 92.6 & 93.9 \\
Finnish & 97.3 & 94.4 & 96.9 \\
French & 98.9 & 96.4 & 99.0 \\
Italian & 99.5 & 98.4 & 98.8 \\
Latvian & 95.7 & 91.0 & 92.1 \\
Lithuanian & 98.8 & 94.6 & 98.2 \\
Polish & 94.9 & 91.1 & 95.2 \\
Russian & 98.6 & 95.4 & 98.2 \\
Slovak & 98.8 & 95.4 & 98.1 \\
Swedish & 98.8 & 96.1 & 94.7 \\
Tamil & 99.3 & 97.0 & 98.3 \\
Ukrainian & 95.8 & 94.6 & 95.9 \\
\hline Average & $\mathbf{9 8 . 2}$ & $\mathbf{9 5 . 1}$ & $\mathbf{9 7 . 0}$ \\
\hline
\end{tabular}

Table 2: Evalutation of the rule-based systems on Gold UD data: Dehouck (Dehouck et al., 2020), Heinecke (Heinecke, 2020) and our system

\subsection{Discrepancies in French}

For the French corpus, we observed 589 discrepancies $^{7}$ of the computed annotation with the gold annotation, and we manually analyzed the first 100 . Table 3 details this analysis.

In order to explain mislabeling in the propagation of incoming dependencies of a coordination, let us return to the example in Figure 2. In propagating incoming dependencies of a coordination that is a modifier, we cannot automatically propagate the label related to the first conjunct to the other conjuncts, because we have to take into account the POS of the heads of these conjuncts. This was not taken into account for the two errors mentioned in the table.

In the table, we have also distinguished errors related to subject or object attributives from errors related to raising and control verbs, because of their particular property: the attributives may have other POS than verb. For exemple, consider the sentence ils laissent les troupes de la KFOR en paix (they leave the KFOR troops in peace). The noun peace has an enhanced subject, which is troops. The gold annotation ignores this type of subject.

44 discrepancies come from the fact that the gold annotation implements enhancements that are not considered by the guidelines. Columns $\neg$ EUD gives a detailed analysis of these enhancements with the number by type. Let us give an example to explain the last type of these non-standard enhancements. Consider the phrase l'occasion également pour J.-P. Bruneau de présenter ses voeux (the occasion also for J.-P. Bruneau to present his wishes). The gold annotation indicates that $J .-P$. Bruneau is the enhanced subject of present, which is not

\footnotetext{
${ }^{7}$ Number of differences computed by the diff Unix tool: differences in consecutive lines are merged as one difference.
} 


\begin{tabular}{|c|c|c|c|c|}
\hline & EUD & UD & GRS & $\neg \mathbf{E U D}$ \\
\hline Total & 47 & 1 & 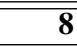 & 44 \\
\hline Non-propagated incoming dependencies of a coordination & 29 & & & \\
\hline Non-propagated left outgoing dependencies of a coordination & 6 & & & \\
\hline Wrongly propagated left outgoing dependencies of a coordination & & & 1 & \\
\hline Non-propagated right outgoing dependencies of a coordination & 2 & & & \\
\hline Label errors in the propagation of incoming dependencies of a coordination & 2 & & & \\
\hline Forgotten subject of controlled verb & 3 & & 1 & \\
\hline Verbs considered as control verbs by error & & & 2 & \\
\hline Forgotten subjects of subject attributes & 3 & & & \\
\hline Forgotten subjects of object attributes & 2 & & & \\
\hline Grammaticaly ill-formed sentence & & & 1 & \\
\hline Incorrect handling of light verb constructions & & & 3 & \\
\hline Subjects of epithet participles & & & & 28 \\
\hline Subjects of modifier infinitive or participial clauses & & & & 14 \\
\hline Subjects of infinitives in constructions NOUN + PREP + NOUN + de + INF & & & & 2 \\
\hline
\end{tabular}

Table 3: Manual inspection of the 100 first discrepancies in French between GOLD $E U D$ and GREW(GOLD $\left.\operatorname{GD}_{U}\right)$. Columns are: errors in EUD gold annotation, errors in UD gold annotation, errors produced by our GRS, EUD gold annotations non described in guidelines.

considered by the guidelines.

\subsection{Discrepancies in English}

For the English test corpus, we observed 815 discrepancies of the computed annotation with the gold annotation, and we also manually inspected the first 100. Table 4 gives a detailed analysis of these errors with their number by type.

\section{Conclusion}

We have observed that many conversion problem arise with the CASEDEPREL layer. This layer is of course highly dependent of the language (because lexical information is used in relation definitions). This prevent the new relation to be universal and we believe that this is counterproductive in the objective of a universal description among a large set of languages.

In this paper, we have proposed a rule-based system for computing EUD annotation from UD. Our raw results are far behind the best systems of the task. This can be explained by the fact that we are dependent of the basic UD annotation provided by another tool. Moreover, the manual inspection we have made shows that, at least on English and French, the GOLD test data used in the task are not error-free and contains several annotations that are not described in the guidelines. We can suspect that this is in favour of the learning based approaches which are designed to adapt to the annotated data, completely ignoring the guidelines.

Despite its weakness, we believe that our system have several benefits:
- It has highlighted some places where the guidelines require precisions, like the presence of several case or mark on the same head;

- It can be used for improving the existing EUD data in the project by identiying annotation error in the current EUD annotations; using a different approach, we can guess that the errors reported will be complementary to the ones that can spotted with other methods;

- Thanks to the modular aspect of the GRS with rules packages adpated to language specificities, it is usable as a starting point for adding EUD annotation layer on languages where there is no such data and where learning based methods cannot be used.

\section{References}

Guillaume Bonfante, Bruno Guillaume, and Guy Perrier. 2018. Application of Graph Rewriting to Natural Language Processing, volume 1 of Logic, Linguistics and Computer Science Set. ISTE Wiley.

Gosse Bouma, Djamé Seddah, and Daniel Zeman. 2020. Overview of the IWPT 2020 shared task on parsing into enhanced Universal Dependencies. In Proceedings of the 16th International Conference on Parsing Technologies and the IWPT 2020 Shared Task on Parsing into Enhanced Universal Dependencies, pages 151-161, Online. Association for Computational Linguistics.

Gosse Bouma, Djamé Seddah, and Daniel Zeman. 2021. From raw text to enhanced universal dependencies: 


\begin{tabular}{|c|c|c|c|c|}
\hline & EUD & UD & GRS & Guidelines \\
\hline Total & 54 & 17 & $\mathbf{1 0}$ & $\mathbf{1 9}$ \\
\hline Non-propagated incoming dependencies of a coordination & 9 & & & \\
\hline Non-propagated outgoing dependencies of a coordination & 18 & & 4 & \\
\hline Propagation of incoming dependencies of a coordination & 7 & & 1 & \\
\hline Verbs considered as control verbs by error & 1 & & & \\
\hline Forgotten subjects of subject attributes & 2 & & & \\
\hline Forgotten subjects of object attributes & 1 & & & \\
\hline Wrongly processed relative clause constructions & 2 & & & \\
\hline Errors in raising of single labels for case or mark dependencies & 11 & & 2 & \\
\hline Errors in raising of fixed labels for case or mark dependencies & 3 & & & \\
\hline Pos extension forgotten in nmod: pos labels & & & 1 & \\
\hline Wrongly processed ellipsis & & & 2 & \\
\hline Relatives pronouns annotated as interrogative & & 17 & & \\
\hline Two case / mark on the same source & & & & 15 \\
\hline Non alphabetic ADP (like"“@”) & & & & 4 \\
\hline
\end{tabular}

Table 4: Manual inspection of the 100 first discrepancies in English between gold annotation and GRS applied on $\mathrm{GOLD}_{U D}$. Columns are: errors in EUD gold annotation, errors in UD gold annotation, errors produced by our GRS, cases unspecified in the guidelines.

The parsing shared task at iwpt 2021. In Proceedings of the 17th International Conference on Parsing Technologies (IWPT 2021), pages 146-157, Bangkok, Thailand (online). Association for Computational Linguistics.

Marie Candito, Guy Perrier, Bruno Guillaume, Corentin Ribeyre, Karën Fort, Djamé Seddah, and Éric de la Clergerie. 2014. Deep syntax annotation of the sequoia French treebank. In Proceedings of the Ninth International Conference on Language Resources and Evaluation (LREC'14), pages 2298-2305, Reykjavik, Iceland. European Language Resources Association (ELRA).

Mathieu Dehouck, Mark Anderson, and Carlos GómezRodríguez. 2020. Efficient EUD parsing. In Proceedings of the 16th International Conference on Parsing Technologies and the IWPT 2020 Shared Task on Parsing into Enhanced Universal Dependencies, pages 192-205, Online. Association for Computational Linguistics.

Johannes Heinecke. 2020. Hybrid enhanced Universal Dependencies parsing. In Proceedings of the 16th International Conference on Parsing Technologies and the IWPT 2020 Shared Task on Parsing into Enhanced Universal Dependencies, pages 174-180, Online. Association for Computational Linguistics.

Joakim Nivre, Marie-Catherine de Marneffe, Filip Ginter, Jan Hajič, Christopher D. Manning, Sampo Pyysalo, Sebastian Schuster, Francis Tyers, and Daniel Zeman. 2020. Universal Dependencies v2: An evergrowing multilingual treebank collection. In Proceedings of the 12th Language Resources and Evaluation Conference, pages 4034-4043, Marseille, France. European Language Resources Association.

Sebastian Schuster and Christopher D. Manning. 2016. Enhanced English Universal Dependencies: An improved representation for natural language understanding tasks. In Proceedings of the Tenth International Conference on Language Resources and
Evaluation (LREC'16), pages 2371-2378, Portorož, Slovenia. European Language Resources Association (ELRA).

Milan Straka, Jan Hajič, and Jana Straková. 2016. UDPipe: Trainable pipeline for processing CoNLL-U files performing tokenization, morphological analysis, POS tagging and parsing. In Proceedings of the Tenth International Conference on Language Resources and Evaluation (LREC'16), pages 42904297, Portorož, Slovenia. European Language Resources Association (ELRA). 